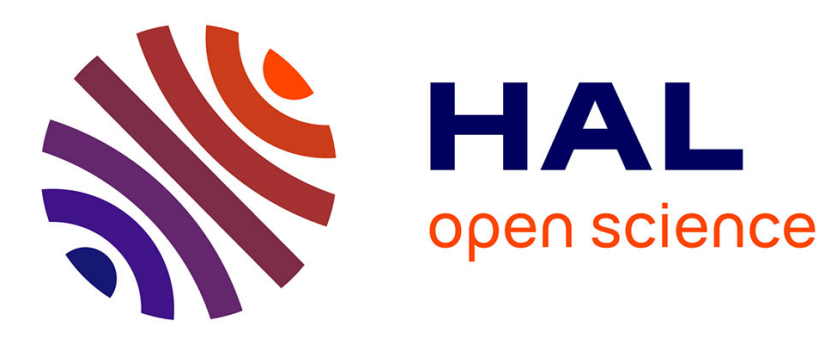

\title{
Self-Turbulent Flame Speeds
}

Joel Quinard, Geoff Searby, Bruno Denet, Jose Graña-Otero

\section{To cite this version:}

Joel Quinard, Geoff Searby, Bruno Denet, Jose Graña-Otero. Self-Turbulent Flame Speeds. Flow, Turbulence and Combustion, 2012, 89 (2), pp.231-247. 10.1007/s10494-011-9350-3 . hal-01315949

\section{HAL Id: hal-01315949 \\ https://hal.science/hal-01315949}

Submitted on 13 May 2016

HAL is a multi-disciplinary open access archive for the deposit and dissemination of scientific research documents, whether they are published or not. The documents may come from teaching and research institutions in France or abroad, or from public or private research centers.
L'archive ouverte pluridisciplinaire HAL, est destinée au dépôt et à la diffusion de documents scientifiques de niveau recherche, publiés ou non, émanant des établissements d'enseignement et de recherche français ou étrangers, des laboratoires publics ou privés. 


\section{Self-turbulent flame speeds}

\section{J. Quinard · G. Searby · B. Denet · J. Graña-Otero}

Received: 14 September 2010 / Accepted:

Abstract This paper reports an experimental investigation of premixed propane and methaneair flames propagating freely in tubes $1.5 \mathrm{~m}$ long and with diameters ranging from 26 to $141 \mathrm{~mm}$. The thermo-acoustic instability was eliminated by means of a novel acoustic absorber placed at the closed end of the tube. We first remark that the flame can adopt different shapes either quasi-axisymmetric and normal to the mean direction of propagation, or inclined with a larger propagation speed because of the increase in flame surface area. The

J. Quinard · G. Searby · B. Denet

CNRS and Aix-Marseille Université,

Institut de Recherche sur les Phénomènes Hors-Equilibre,

49 rue F. Joliot Curie, F-13384 Marseille, France

Corresponding author

Tel: +(33) 496139721

Fax: +(33) 496139709

E-mail: : quinard@irphe.univ-mrs.fr

J. Graña-Otero

Thermodynamics Departement at ETSI Aeronauticos

Universidad Politécnica de Madrid, Spain 
minima of the propagation speeds, corresponding to non-tilted flame propagation, are then analyzed using analytical models for the self-turbulent flame propagation. The concept of a cut-off wavelength appears to be relevant to explain the different behaviors observed on the rich side of methane-air and propane-air flames.

Keywords Premixed combustion · Self-turbulent flame speed · Flame instability

\section{Introduction}

Since the pioneering work of Mallard and Le Chatelier [1], who determined the flammability limits and the propagating velocity of various combustible gaseous mixtures, there have been numerous studies concerning the prediction of turbulent flames speeds and safety limits. The result is generally written as

$$
U_{T} / U_{L}=1+\left(u^{\prime} / U_{L}\right)^{\alpha},
$$

where $U_{T}$ is the turbulent flame speed, $U_{L}$ the laminar flame speed, $u^{\prime}$ the r.m.s. turbulent velocity of the incoming flow and $\alpha$ an exponent whose value varies between 0.5 and 2 depending on the modeling assumptions [2-4]. The low turbulence limit would thus be $U_{T} \approx U_{L}$. However it is well-known that laminar flames propagating freely in quiescent mixtures propagate at higher velocities due to curvature effects induced by hydrodynamic and possibly thermo-diffusive instabilities [5-7]. There have been already attempts to incorporate flame instabilities into such a formula $[8,9]$ and the very existence of such an universal formula has been questioned recently on the basis of both experiments and numerical modeling $[10,11]$. Assuming a semi-cylindrical or hemispherical shape for the cells on the flame in a 2-D or 3-D configuration, the ratio $U_{T} / U_{L}$ would tend to $\pi / 2$ or 2 respectively. These values are similar to the results of recent analytical and numerical investigations for flame 
propagation in narrow tubes that suggest that the maximum velocity ratio is approximately 1.3 for two-dimensional flames, and approximately 1.7 in the three-dimensional case.

These investigations were limited to the case of tubes with an inner diameter smaller than $4 \lambda_{c}$, where $\lambda_{c}$ is the cut-off wavelength for flame front instability $[8,12-16]$. For tubes of larger diameter, secondary instabilities occur, possibly leading to a self-similar behavior of the topology of the flame. Using a fractal dimension obtained for expanding spherical flames, a corresponding power-law behavior for the flame propagation speed has been proposed [15]:

$$
U_{T} / U_{L}=\left(\Lambda_{m} / \lambda_{c}\right)^{D}
$$

where $\Lambda_{m}$ is the largest characteristic length of the flame and $D \approx 1 / 3$ is an exponent resulting from the fractal dimension of the flame $[8,17,18]$. This relation seems to work well with weakly turbulent premixed flames $[4,12]$, but to the best of our knowledge, apart from the ancient experimental observations of Coward \& Hartwell [19] performed in large horizontal tubes where buoyancy effects are important, such an increase of the flame speed with the tube radius has never been seen experimentally for flames propagating freely in a quiescent gas. One probable reason is the occurrence of violent thermo-acoustic instabilities that completely change the shape and propagation velocity $[20,21]$ of flames propagating in tubes.

In this paper, our objective is to observe the dependence of the propagation speed of cellular flames in a quiescent medium on the characteristic dimensions of the burner. In the next section we will briefly describe the very "simple" experiment. The results obtained with propane- and methane-air flames will then be presented and discussed in the last section with some comments on the way to determine the characteristic lengths $\Lambda_{m}$ and $\lambda_{c}$. 


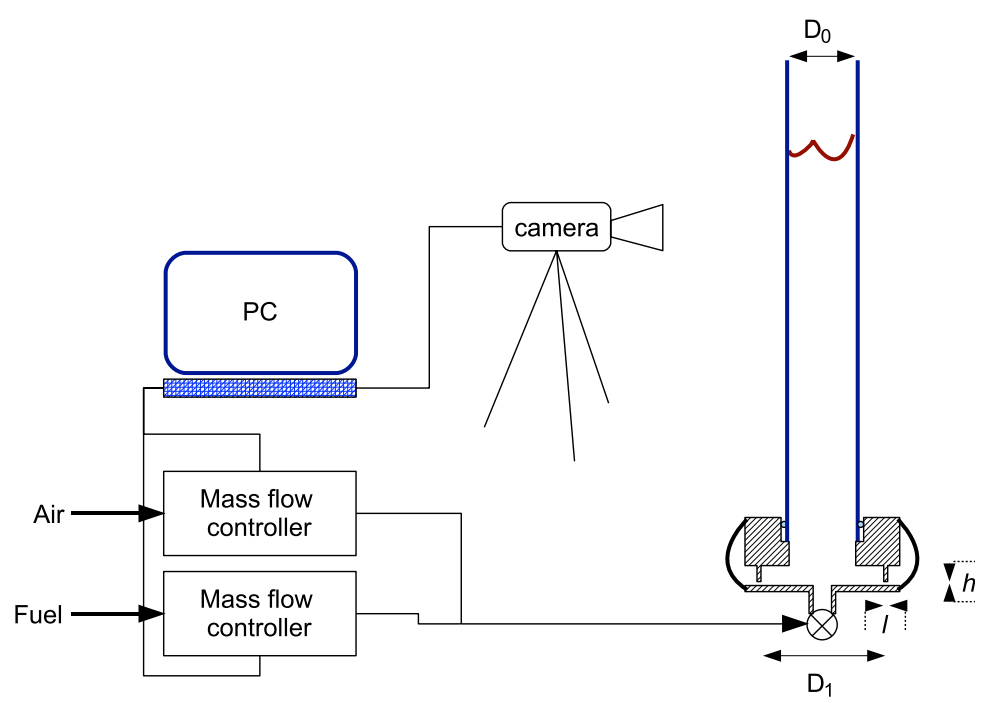

Fig. 1 Schematic of the experimental arrangement. The dimensions $D_{0}, D_{1}, l, h$ are explained in the text.

\section{Experimental setup and procedure}

The propagation velocity of laminar cellular flames is measured in a vertical Pyrex tubes, $1.5 \mathrm{~m}$ long, with internal diameters ranging from 26 to $141 \mathrm{~mm}$. The equivalence ratio, $\phi$, of the premixed gas is controlled via a PC-interface connected to mass-flow regulators. The flame propagation is recorded using a video camera. The particularity of this experiment is related to the bottom part of the burner where an acoustic damper is installed to prevent the onset of thermo-acoustic instabilities. The damper consists of a small annular slit, of height $h$ and length $l$ that dissipates acoustic energy by terminating the tube with a real (resistive) acoustic impedance equal to the characteristic acoustic impedance of free air. The details of the damper are given in the appendix. An expansion chamber is placed outside of the slit to avoid mixing of ambient air with the combustible mixture.

The operating procedure is as follows: After each run, the air flow is opened and maintained until the tube walls have cooled to ambient temperature. The flow of combustible, methane or propane, is then adjusted to the desired equivalence ratio and a lightweight plate 
is placed over the open end of the burner. The presence of the plate is sufficient to prevent mixing with ambient air, but does not prevent exhaust of the premixed gas. The flow is maintained for a time corresponding to at least ten fillings of the tube and then stopped by closing the valve at the bottom of the burner. A delay of at least one minute is allowed before gently withdrawing the upper plate and igniting the mixture with a lighter.

The video-movie is then digitised and post-processed using ImageJ 1.40 software to obtain the trajectory of the upstream tip of the flame.

\section{Results}

The first experimental result is that a given flame does not propagate systematically at a fixed speed, even if the measured velocity can be constant during all or part of a given experiment (see fig. 2, left line). Another experiment made in the same conditions with the same equiv-

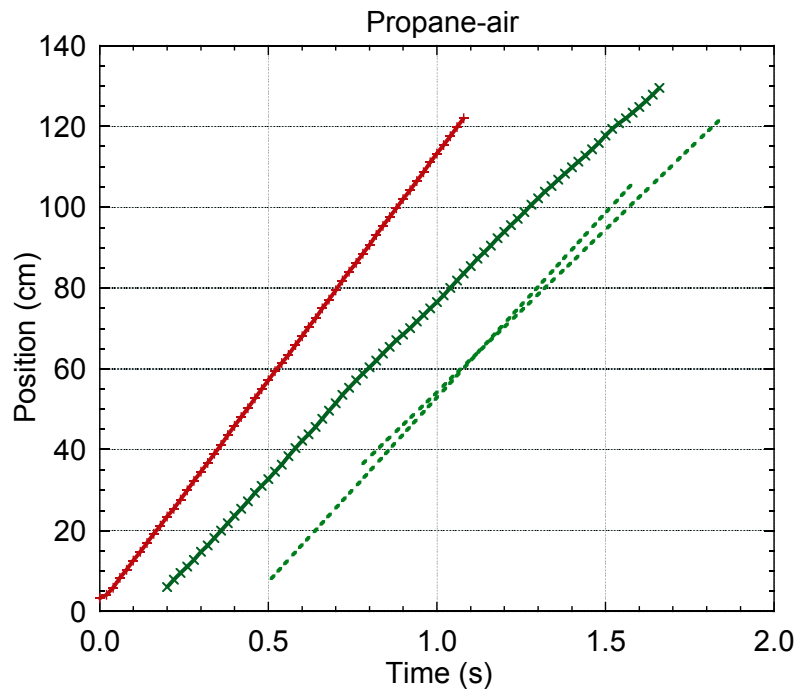

Fig. 2 Record of two propane-air flame trajectories in the same tube, diameter $90 \mathrm{~mm}$, equivalence ratio 0.9 . The dotted lines are parallel to the experimental points of the trajectory on the right to highlight the change in flame speed. 


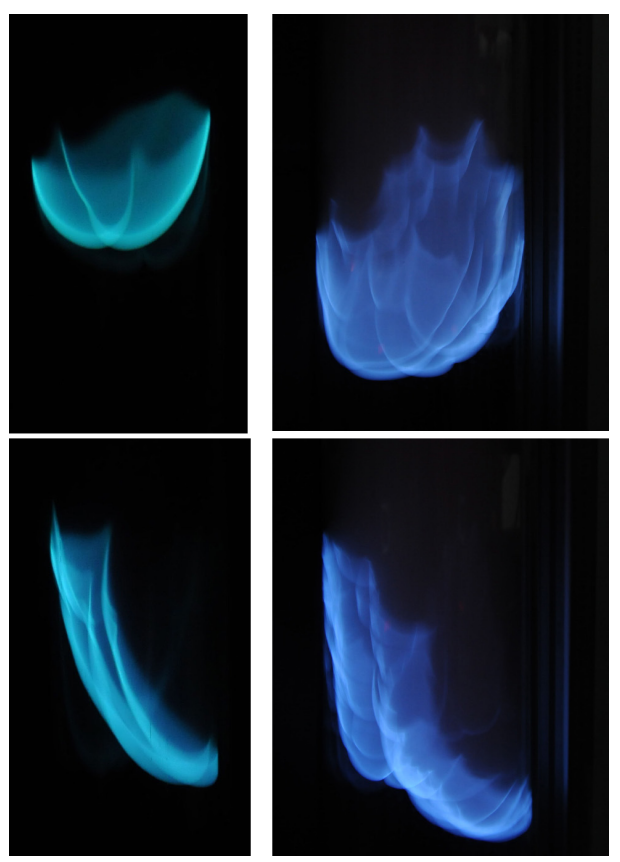

Fig. 3 Different shapes of propane-air flames during free propagation in tubes. Left: $\phi=1.2, \varnothing$ tube $=$ $40 \mathrm{~mm}$; Right: $\phi=0.9, \varnothing$ tube $=140 \mathrm{~mm}$ (not the same scale).

alence ratio, $\phi=0.9$, shows the flame initially propagating at a slightly smaller speed and then decelerating suddenly (at $t \approx 0.8 \mathrm{~s}$ ) to reach a still smaller speed of propagation (see fig. 2, right line). We find that there is almost a continuum of flame speeds related to different flame shapes, see fig. 3 .

It has been found theoretically from the Sivashinsky equation [22] that multiple stationary solutions exist for the problem of a flame propagating in a (2-D) tube, including the two typical flame shapes similar to those we observe in our experiments: a flame inclined relative to its direction of propagation (fig. 3, bottom), or a slightly asymmetric flame (fig. 3, top). These two different type of solutions have also been found in direct 2-D numerical simulations or potential models $[8,12,13,23]$, with a velocity increase ranging from $1.3 U_{L}$ to $1.58 U_{L}$. In our experiments we find larger flame velocities, in the range from $1.5 U_{L}$ to 

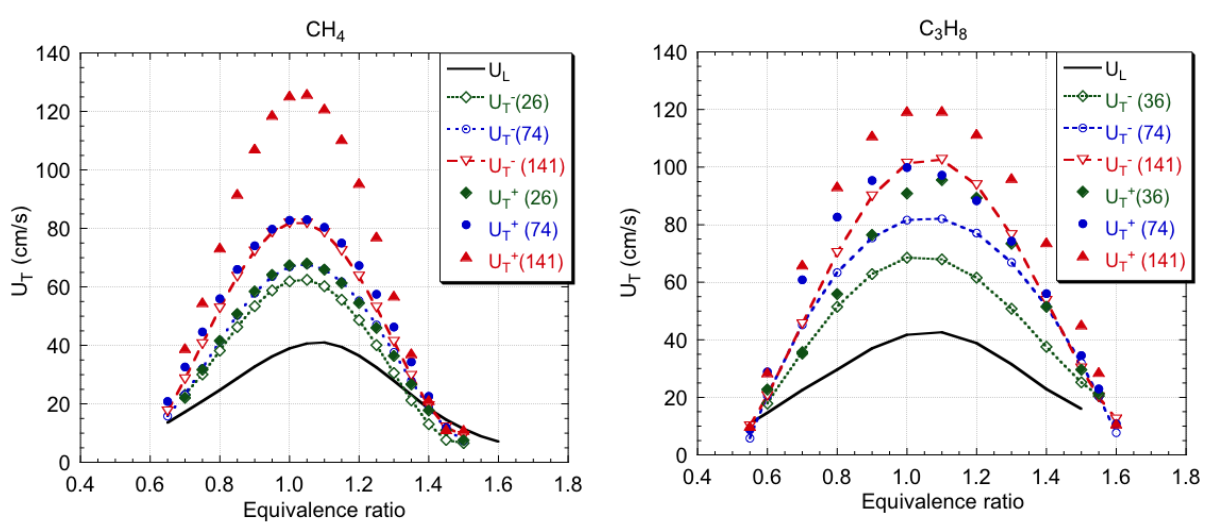

Fig. 4 Minimum $\left(U_{T}^{-}\right.$: dotted lines with open symbols) and maximum ( $U_{T}^{+}$: solid symbols) methane-air (left) and propane-air (right) self-turbulent flame speed in tubes of different diameter. $U_{L}$ is the laminar flame speed. The numerical values in the legend give the tube diameter in $\mathrm{mm}$.

$3 U_{L}$ see fig. 4 , but it is generally recognized [24] that the velocity increase relative to the laminar velocity is higher in 3-D than in 2-D. Another important result is that the inclined flame velocity is larger than the slightly asymmetric one, a property which is not found in the Sivashinsky equation [22], where these two solutions have the same velocity, but which is found in a potential model [23]. Increased velocity of inclined flames has been also demonstrated in direct numerical simulations of flame dynamics[12].

It is generally believed that the fastest solution should dominate at long times, however this is not clear from our experiments since both slow to fast and fast to slow transitions were observed, with either a quasi-axisymmetric flame evolving into an inclined flame, or an inlined flame evolving into a flame normal to the mean direction of propagation. The fact that both slow to fast, and fast to slow transitions can be observed for flames propagating in the same mixture and in the same tube indicates that: a) the ignition protocol is not the (only) factor controlling the onset of slanted propagation, and b) this transition is very sensitive to very small perturbations. 
The results in figure 4 present the maxima $\left(U_{T}^{+}\right)$and minima $\left(U_{T}^{-}\right)$of the self-turbulent flame speed in tubes of different diameters. They were obtained from 2 to 4 runs per configuration, retaining only events where the flame was propagating uniformly during typically $30 \%$ of the tube length, and then adjusting two curves to interpolate between the extrema of the measured values. In some cases the flame was always inclined, whereas in some other cases, it was only weakly tilted. More experiments are thus needed, and probably with a more sophisticated and reproducible igniter, to reproduce the whole range of possible flame velocities. Nevertheless the range of flame speed spanned by this set of experiments is wide enough to distinguish clearly the two limits, the scatter of the experimental measurements on the curve-fits being less than $10 \%$.

The reference speed $U_{L}$ is taken from measurements of Bosshaart \& De Goey [25] which were found to be very close to our own measurements on some planar flames in tubes. The general trends seem to be the same for both propane- and methane-air flames: the increase in flame speed near to the extinction limits is only small: for slow flames the stabilising effect of gravity is important and forces the flame to be almost flat. For faster flames, in the midrange of equivalence ratios, both the maximum and the minimum flame speeds increase with tube diameter with no indication of any saturation effect in the largest tube investigated here (141 mm diameter).

\section{Analysis}

4.1 Reduced results:

Retaining only the minima of the measured flame speeds, $U_{T}^{-}$, corresponding to non-tilted flame propagation, the results are presented in figure 5 as the normalised velocity ratio $U_{T}^{-} / U_{L}$. For methane-air flames, there is no dramatic change of the flame speed when the 

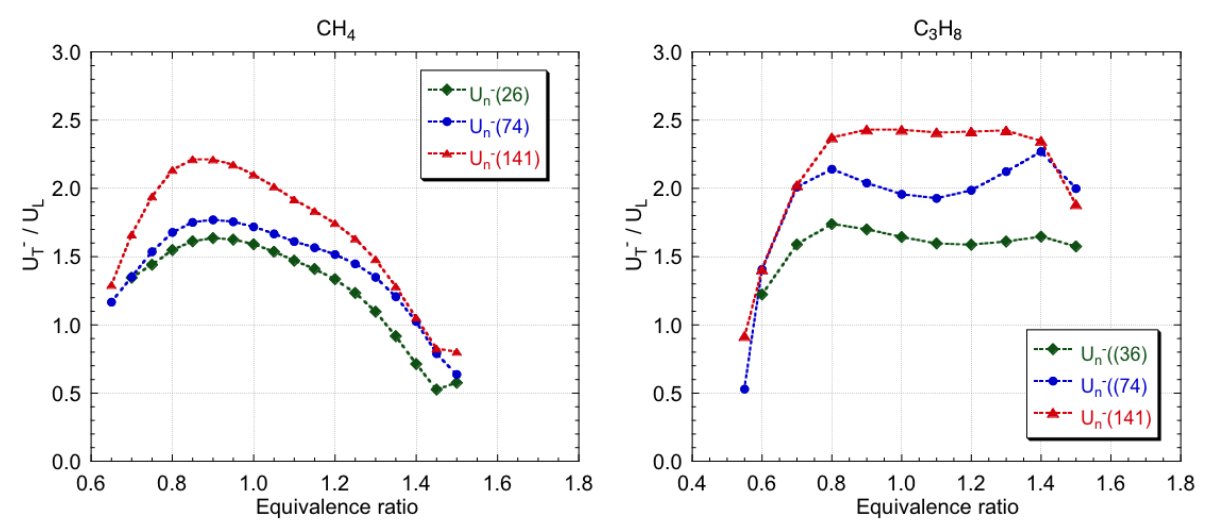

Fig. 5 Normalized velocity ratio of methane-air (left) and propane-air (right) flame speed in tubes of different diameter. The numerical values in the legend give the tube diameter in $\mathrm{mm}$

tube diameter is increased from 26 to $74 \mathrm{~mm}$. The only significant increase in normalised flame speed occurs for methane flames in the $141 \mathrm{~mm}$ tube. It will be seen later that the largest tube is the only one for which the cut-off wavelength of methane flames is very much smaller than the tube diameter. However it is also difficult to exclude a bias due to the fact that it is very difficult to obtain a non-tilted fast methane flame in the smaller tubes, which have a larger Froude number for the same flame speed. phrase à supprimer :The fact that the minimum velocity is not always measured is also obvious when considering the crossing of the two curves with tube diameters respectively of 55 and $74 \mathrm{~mm}$. Nevertheless all these curves present a maximum on the lean side, the maximum velocity being $2.2 U_{L}$ in the $141 \mathrm{~mm}$ tube near $\phi \approx 0.8$. This value is also close to the normalised burning velocity that can be extrapolated to $u^{\prime} / U_{L}=0$ from the experimental data in reference [4].

The normalised velocity ratio drops to a value even less than unity for the richest flames, $\phi \geq 1.4$, showing that heat loss effects are significantly strong for these very slow flames (see also figure 4). 
For propane-air flames, the increase in flame speed with the tube diameter is much more regular. There is a maximum on the lean side near to $\phi \approx 0.8$, but there is also a second maximum on the rich side and the maximum velocity ratio in the largest tube reaches the value $\approx 2.5$ over a large range of equivalence ratios from $\phi=0.8$ to $\phi=1.4$. The curves are truncated at $\phi=1.5$ because the value of the laminar flame speed, $U_{L}$ is not given in ref. [25] for equivalence ratios beyond 1.5. However it is clear from fig. 4 that the limiting value of the velocity ratio will be close to unity.

The reason for the difference in behaviour of rich methane and propane flames is clearly seen in fig. 6. Rich methane-air flame have large relatively smooth cells, whereas the rich propane-air flame have numerous secondary cells superimposed on the larger cells, leading to a greater increase in flame surface area. A usual property of the hydrodynamic instability is that small cells are convected towards the cusps of the larger cells. They finally merge with the cusp, thus increasing the amplitude of the larger cell. However for large tubes (compared to $\lambda_{c}$ ) the front is very sensitive to any form of noise in the system (here residual turbulence), and new small cells are continuously created on the front. A WKB argument [26] suggests
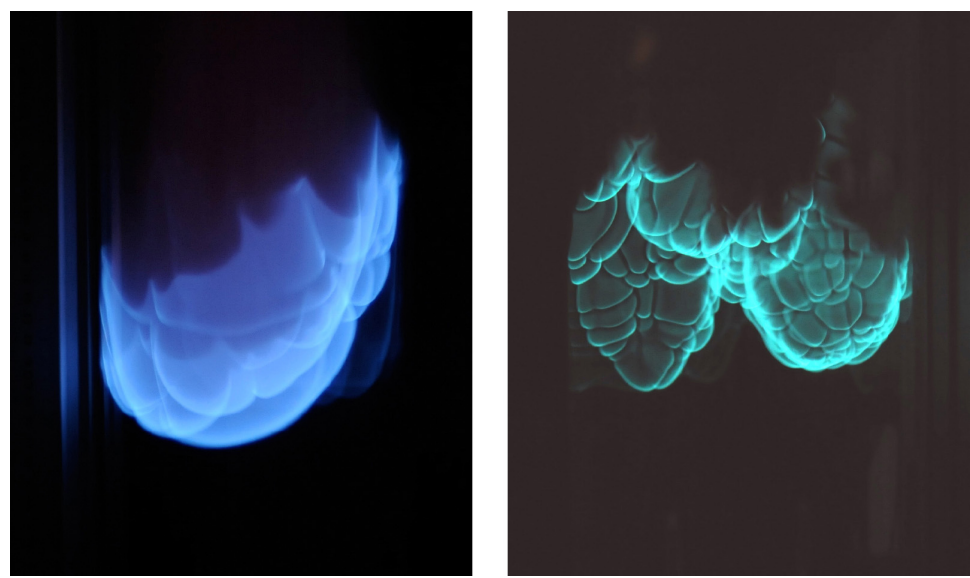

Fig. 6 Rich methane-air (left: $\phi=1.3$ ) and propane-air flame (right: $\phi=1.4$ ) in a tube $140 \mathrm{~mm}$ inner diameter. 
that the order of magnitude of these small cells is the most amplified wavelength (approximately $2 \lambda_{c}$ ), so that a smaller $\lambda_{c}$ leads to the creation of smaller new cells. Furthermore, if simulations of the Sivashinsky equation are to be believed [22], for wide tubes (versus $\lambda_{c}$ ), very complicated stationary solutions exist, which could be close to the rich propane-air flame shown in figure 6.

The increase in flame speed in wide tubes is thus related to the cellular instability as already proposed by several authors [8, 12-16]. Akkerman \& Bychkov [15] used measurements of the Markstein lengths taken from the literature [27] to calculate the cut-off wavelengths and to determine flame propagation speeds resulting from the effect of the Darrieus-Landau instability. This set of Markstein numbers for propane and methane-air flames was chosen because it was obtained at the stability limit of planar premixed flames propagating downwards, in accordance with theoretical simplified models used in their numerical approach. However further work [28] demonstrated that this way of determining the Markstein number is valid only when the Lewis number is very close to one. Moreover this work also showed that good agreement between different experimental measurements of Markstein numbers can be obtained only by correct extrapolation of the flow velocity to the reaction zone. This work also demonstrated the necessity of application of a corrective factor when the gas velocity is extrapolated from the burnt gas side, as for instance, when using measurements on spherically expanding flames.

The value of the Markstein number for different mixture is still a subject of controversy and it is thus interesting to compare relation (2) using different ways to calculate the characteristic lengths $\Lambda_{m}$ and $\lambda_{c}$. 
4.2 Characteristic lengths of unstable flames

It can be assumed that the characteristic lengths $\Lambda_{m}$ and $\lambda_{c}$ are related to the extrema of unstable wavelengths of a reactive mixture determined from linear theory. These one are given by the roots of the dispersion relation, which in turn depends on the Markstein number, Ma, of the mixture. In the following we will compare three different ways to obtain Markstein number. These three estimation of Ma will be denoted by Ma I, Ma II and Ma III respectively.

According to analytical calculations of the stability of premixed planar flames including expansion effects, gravity and preferential diffusion $[2,29]$, the rate of growth, $\sigma$, of small perturbations with a wavenumber $k=2 \pi / \Lambda$ is given by

$$
\sigma=k U_{L} \Omega
$$

with

$$
\Omega=\frac{E}{E-1}\left\{\left[\frac{E^{2}+E-1}{E}+\operatorname{Ma} d_{L} k\left(\operatorname{Ma} d_{L} k-2 E\right)-\frac{E^{2}-1}{E^{2}} \frac{g}{k U_{L}^{2}}\right]^{1 / 2}-\operatorname{Ma} d_{L} k-1\right\},
$$

where $E$ is the expansion ratio $\rho_{u} / \rho_{b}$, Ma is the Markstein number, $d_{L}$ the laminar flame thickness and $g$ the acceleration of gravity. The subscripts $u$ and $b$ refer respectively to unburnt and burnt gases.

For downward propagating flames above the threshold of cellular instability,

$$
U_{L}>\sqrt{8 \mathrm{Ma} d_{L} g /(E-1)}
$$

there is a band of unstable wavelengths limited by two neutral wavenumbers:

$$
k_{n}^{ \pm}=\frac{E-1}{4 E \operatorname{Mad} d_{L}}\left(1 \pm \sqrt{1-\frac{8 \mathrm{Ma} d_{L} g}{(E-1) U_{L}^{2}}}\right) .
$$


At the threshold of stability for a planar flame, $k_{n}^{+}=k_{n}^{-}=2 \pi / \Lambda_{M}^{*}$ where $\Lambda_{M}^{*}$ is the most unstable wavelength, and the Markstein number for these mixtures can be determined experimentally in two ways:

- either by using the critical flame speed $U_{L}^{*}$ at the stability limit as was done in [27]:

$$
\mathrm{Ma}^{*} \mathrm{I}=\frac{\left(E^{*}-1\right) U_{L}^{* 2}}{8 g d_{L}^{*}}
$$

- or by using the critical wavelength measured at the stability limit

$$
\mathrm{Ma}^{*} \mathrm{II}=\frac{\left(E^{*}-1\right)}{8 \pi E^{*}} \frac{\Lambda_{M}^{*}}{d_{L}^{*}}
$$

In the above expressions, the superscript * denotes values at the threshold of stability.

The second relation has never been used because the published results are relatively scarce $[30,31]$, but it can be expected to be more appropriate to the present problem since it is directly related to the cell size.

However, it is known that changes in the gas expansion ratio can affect the value of the Markstein number. Since the stability limits of planar flames were measured using diluted flames with low flame speeds and small expansion ratios, and our measurements were performed for non-diluted flames over a large range of flame speeds and expansion ratios, the differences in expansion ratio must be taken into account in the evaluation of the characteristic lengths of the flame. Assuming a hard-sphere model for the gas mixture and a one-step irreversible Arrhenius reaction, Clavin and Garcia [32] obtained the following analytical expression for the Markstein number:

$$
\mathrm{Ma}=\frac{2 E}{\sqrt{E+1}}+\beta(\mathrm{Le}-1)\left[\frac{2}{\sqrt{E+1}}-\frac{E}{E-1} \ln \left(\frac{\sqrt{E}+1}{2}\right)\right]
$$

where Le is the Lewis number of the limiting reactant and $\beta$ is the Zeld'ovich number. This expression can be used to correct the Markstein number for flames with the same equivalence ratio (and Lewis number), but different expansion ratios (dilutions). Knowing 
the Markstein number at the threshold of stability, the reduced Lewis number $\beta(L e-1)$ can be determined from (8) to calculate ultimately the Markstein number with the actual expansion ratio E from GASEQ [33]. The Markstein numbers obtained from experimental measurements of the instability threshold, using relations (6) and (7), corrected using (8), will be called Ma I and Ma II respectively.

Finally, some values of Markstein numbers obtained by direct numerical simulations of stretched methane and propane flames with detailed chemical kinetics are available in the literature [28]. These values from numerical simulation will be called Ma III.

Tables I and II resume the parameters we have used to calculate $\lambda_{c}=2 \pi / k^{+}$and $\Lambda_{m}=$ $2 \pi / k^{-}$in order to test equation (2). The flame thickness is taken equal to $D_{t h} / U_{L}$ with $D_{t h}=$ $0.2 \mathrm{~cm}^{2} \mathrm{~s}^{-1}$

In these tables, $U_{L}^{*}, \Lambda_{M}^{*}$ and the expansion ratio at the threshold of instability, $E^{*}$, are taken from [30, 31]. They are used to determine $\mathrm{Ma}^{*}$ from (6) (7), and the effective value of $\beta($ Le-1) in (8). Rem: il y a redite, doit-on la supprimer ?

It can be seen that the Markstein numbers calculated using equations (6) and (7) are very close so, for the sake of clarity, only Ma II and Ma III (from ref [28]) will be used to calculate the characteristic velocities of the flames. The values of $\lambda_{c}=2 \pi / k_{n}^{+}$and $\Lambda_{m}=$ $2 \pi / k_{n}^{-}$are then calculated using equ. (5). The resulting values are plotted in figure 7 . The longest unstable wavelength, $\Lambda_{m}$, has a maximum value close to $\phi \approx 1.1$ and decreases towards the extinction limits. $\Lambda_{m}$ is larger for propane than for methane/air flames as a result of a larger laminar flame speed on one part, and because preferential diffusion is less stabilizing for the rich propane-air flames. In general, the long wavelength cut-off, $\Lambda_{m}$, is larger than the diameter of the tube, except for slow flames in large tubes.

For methane flames, the short wavelength cut-off, $\lambda_{c}$, has a minimum value around stoichiometry with an order of magnitude of 0.5 to $1 \mathrm{~cm}$. The range of unstable wavelengths 
Table 1 Parameters used to calculate characteristic lengths of methane-air flames (units: cm, s).

Labels: '*'= results from [31]; 'I'= equ. (6); 'II'= equ. (7); 'III'= results from [28].

\begin{tabular}{|c|c|c|c|c|c|c|c|c|c|c|c|}
\hline$\phi$ & $U_{L}^{*}$ & $\Lambda_{M}^{*}$ & $E^{*}$ & $\begin{array}{l}\mathrm{Ma}^{*} \mathrm{I} \\
\left(U_{L}^{*}\right)\end{array}$ & $\begin{array}{c}\mathrm{Ma}^{*} \mathrm{II} \\
\left(L^{*}\right)\end{array}$ & $\begin{array}{c}E \\
\mathrm{CH}_{4} \text {-air }\end{array}$ & Ma I & Ma II & Ma III & $\begin{array}{c}U_{L} \\
\mathrm{CH}_{4} \text {-air }\end{array}$ & $\begin{array}{l}d_{L} \\
\mathrm{CH}_{4} \text {-air }\end{array}$ \\
\hline 0.560 & 8.40 & 1.41 & 5.60 & 1.74 & 1.93 & 5.30 & 1.55 & 1.74 & - & 6.00 & 0.0333 \\
\hline 0.600 & 9.30 & 1.53 & 5.70 & 2.41 & 2.34 & 5.55 & 2.32 & 2.25 & 2.98 & 7.89 & 0.0253 \\
\hline 0.700 & 9.50 & 1.57 & 5.90 & 2.68 & 2.46 & 5.55 & 2.48 & 2.26 & 3.64 & 15.1 & 0.0133 \\
\hline 0.800 & 9.80 & 1.64 & 5.90 & 2.94 & 2.65 & 6.65 & 3.32 & 3.05 & 4.69 & 23.6 & 0.00847 \\
\hline 0.900 & 10.0 & 1.69 & 6.00 & 3.19 & 2.79 & 7.11 & 3.73 & 3.36 & 5.54 & 31.3 & 0.00639 \\
\hline 0.950 & 10.2 & 1.74 & 6.10 & 3.45 & 2.95 & 7.30 & 4.01 & 3.55 & - & 33.9 & 0.00589 \\
\hline 0.980 & 10.4 & 1.80 & 6.10 & 3.65 & 3.11 & 7.38 & 4.24 & 3.73 & - & 35.6 & 0.00562 \\
\hline 1.000 & 10.6 & 1.86 & 6.10 & 3.87 & 3.28 & 7.42 & 4.46 & 3.91 & 6.20 & 36.3 & 0.00551 \\
\hline 1.020 & 10.8 & 1.93 & 6.10 & 4.09 & 3.46 & 7.44 & 4.67 & 4.08 & - & 36.8 & 0.00544 \\
\hline 1.050 & 11.0 & 2.00 & 6.10 & 4.32 & 3.65 & 7.44 & 4.89 & 4.26 & - & 37.0 & 0.0054 \\
\hline 1.100 & 11.1 & 2.03 & 6.00 & 4.36 & 3.74 & 7.37 & 4.93 & 4.36 & 6.99 & 37.0 & 0.00541 \\
\hline 1.200 & 11.2 & 2.10 & 5.90 & 4.39 & 3.89 & 7.12 & 4.90 & 4.43 & 7.96 & 33.9 & 0.00589 \\
\hline 1.300 & 11.3 & 2.10 & 5.90 & 4.50 & 3.92 & 6.86 & 4.91 & 4.35 & 9.13 & 26.3 & 0.0076 \\
\hline 1.400 & 11.5 & 2.20 & 5.80 & 4.65 & 4.17 & 6.60 & 4.98 & 4.52 & 6.73 & 17.5 & 0.0115 \\
\hline 1.500 & 12.3 & 2.70 & 5.70 & 5.57 & 5.45 & 6.35 & 5.81 & 5.69 & - & 11.3 & 0.0177 \\
\hline
\end{tabular}

is only a factor $\approx 2$ for slow flames in small tubes, and $\approx 30$ for stoichiometric flames in the widest tube. Model Ma III predicts cut-off wavelengths that are about 50\% larger than Ma II, with a corresponding decrease in the range of unstable wavelengths.

For propane flames, models Ma I and Ma II predict a short wavelength cut-off that is quite large $(\approx 1.5 \mathrm{~cm})$ for very lean flames, decreasing rapidly to a very small or zero value for rich flames. This is consistent with the observation that rich propane flames have many small cells on the flame front. It results from the decrease of the Lewis number as the oxygen becomes the limiting reactant so that all flames with $\phi>1.1$ were intrinsically unstable even 
Table 2 Parameters used to calculate characteristic lengths of propane-air flames (units: cm, s).

Labels: '*'= results from [31]; 'I'= equ. (6); 'II'= equ. (7); 'III'= results from [28].

\begin{tabular}{|c|c|c|c|c|c|c|c|c|c|c|c|}
\hline$\phi$ & $U_{L}^{*}$ & $\Lambda_{M}^{*}$ & $E^{*}$ & $\begin{array}{l}\mathrm{Ma}^{*} \mathrm{I} \\
\left(U_{L}^{*}\right)\end{array}$ & $\begin{array}{c}\mathrm{Ma}^{*} \mathrm{II} \\
\left(L^{*}\right)\end{array}$ & $\begin{array}{c}E \\
\mathrm{C}_{3} \mathrm{H}_{8} \text {-air }\end{array}$ & Ma I & Ma II & Ma III & $\begin{array}{c}U_{L} \\
\mathrm{C}_{3} \mathrm{H}_{8} \text {-air }\end{array}$ & $\begin{array}{l}d_{L} \\
\mathrm{C}_{3} \mathrm{H}_{8} \text {-air }\end{array}$ \\
\hline 0.550 & 12.3 & 2.91 & 5.50 & 5.34 & 5.82 & 5.36 & 5.28 & 5.77 & - & 10.0 & 0.0200 \\
\hline 0.600 & 11.7 & 2.38 & 5.50 & 4.59 & 4.53 & 5.67 & 4.66 & 4.61 & 8.80 & 12.6 & 0.0159 \\
\hline 0.700 & 11.5 & 2.23 & 5.56 & 4.42 & 4.19 & 6.27 & 4.72 & 4.50 & 8.00 & 20.7 & 0.00968 \\
\hline 0.800 & 11.4 & 2.16 & 5.60 & 4.34 & 4.03 & 6.81 & 4.86 & 4.57 & 7.75 & 29.8 & 0.00670 \\
\hline 0.900 & 11.2 & 2.03 & 5.65 & 4.16 & 3.73 & 7.27 & 4.86 & 4.46 & 7.75 & 37.6 & 0.00532 \\
\hline 0.950 & 10.8 & 1.81 & 5.70 & 3.77 & 3.20 & 7.44 & 4.55 & 4.04 & - & 40.3 & 0.00496 \\
\hline 1.000 & 10.4 & 1.62 & 5.75 & 3.40 & 2.77 & 7.56 & 4.25 & 3.68 & 7.60 & 42.1 & 0.00475 \\
\hline 1.020 & 10.1 & 1.51 & 5.70 & 3.09 & 2.50 & 7.59 & 4.00 & 3.47 & - & 42.6 & 0.00469 \\
\hline 1.050 & 9.60 & 1.35 & 5.50 & 2.54 & 2.12 & 7.60 & 3.61 & 3.24 & - & 42.9 & 0.00467 \\
\hline 1.080 & 8.60 & 1.16 & 5.40 & 1.78 & 1.62 & 7.58 & 2.99 & 2.85 & - & 42.8 & 0.00467 \\
\hline 1.090 & 7.50 & 1.07 & 5.40 & 1.18 & 1.30 & 7.57 & 2.46 & 2.57 & - & 42.7 & 0.00468 \\
\hline 1.095 & 6.50 & 1.05 & 5.30 & 0.752 & 1.10 & 7.56 & 2.14 & 2.44 & - & 42.6 & 0.00469 \\
\hline 1.096 & 6.00 & 1.04 & 5.20 & 0.578 & 1.01 & 7.56 & 2.05 & 2.42 & - & 42.6 & 0.00469 \\
\hline 1.098 & 3.80 & 1.02 & 5.20 & 0.147 & 0.621 & 7.55 & 1.67 & 2.08 & - & 42.6 & 0.00470 \\
\hline 1.100 & - & - & - & - & - & 7.55 & - & - & 7.04 & 42.5 & 0.00470 \\
\hline 1.200 & - & - & - & - & - & 7.34 & - & - & 5.96 & 38.8 & 0.00515 \\
\hline 1.300 & - & - & - & - & - & 7.08 & - & - & 4.40 & 31.6 & 0.00632 \\
\hline 1.400 & - & - & - & - & - & 6.83 & - & - & 3.14 & 22.6 & 0.00886 \\
\hline 1.500 & - & - & - & - & - & 6.58 & - & - & 2.00 & 14.0 & 0.0143 \\
\hline 1.600 & - & - & - & - & - & 6.34 & - & - & 2.14 & 9.20 & 0.0217 \\
\hline
\end{tabular}

at the lowest flame speed attainable in the experiments of [27][30]. This is why the further increase of the cut-off wavelength is not evaluated from these measurements. Model Ma III predicts a slightly greater value for $\lambda_{c}$ that remains positive for all equivalence ratios, with a minimum value of $\approx 0.4 \mathrm{~cm}$ at an equivalence ratio of 1.4 . According to Ma III the range 

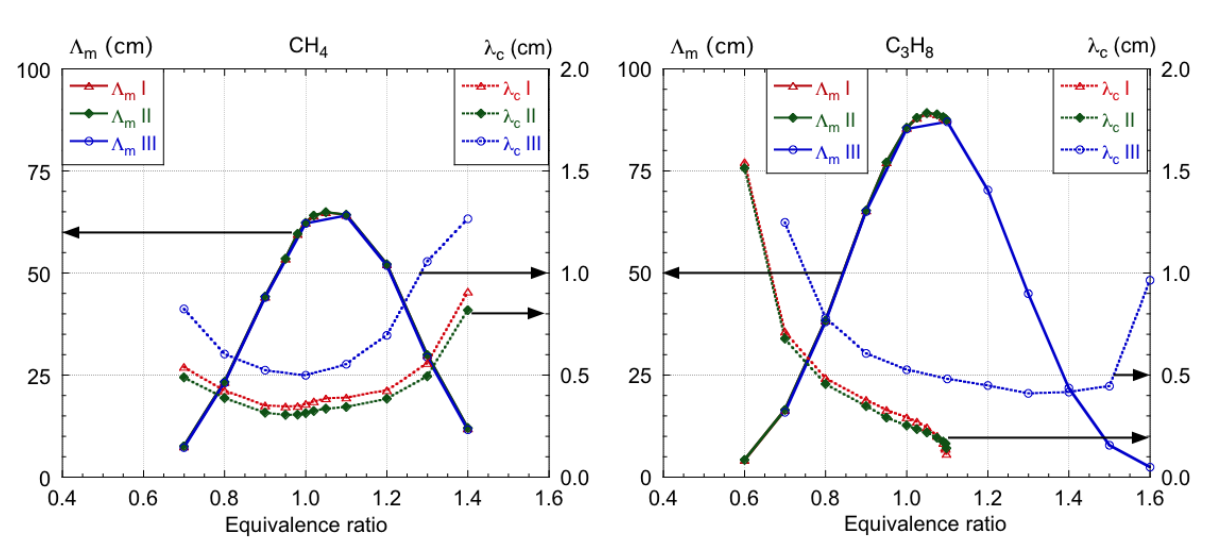

Fig. 7 Longest, $\Lambda_{m}$, and shortest, $\lambda_{c}$, unstable wavelengths calculated for methane and propane flames using equ. (5) for the three different methods of evaluating the Markstein length.

of unstable wavelengths varies from $\approx 1.5$ for lean propane flames in the smallest tube, to $\approx 35$ for rich propane flames in the largest tube.

We should remark that the values Ma III are obtained from numerical measurements of the speed of stretched planar flames in a divergent flow, whereas the values Ma II were obtained from the stability limits in a uniform flow. There is numerical and experimental evidence that the Markstein numbers of curved and stretched flames can be different, [3437].

The resulting normalised turbulent flame speeds are then calculated using equ. (2) with $D=1 / 3$. When the diameter of the tube is smaller than the calculated value of $\Lambda_{m}$ then we have used the former as the upper limit for $\Lambda_{m}$. The results are shown in figure 8 .

The agreement between experimental results and calculated values is not perfect, but the general trends are relatively well reproduced. All curves decrease towards unity near the extinction limits, reflecting the reduced range of unstable wavelengths.

The self-similar flame velocity calculated using Ma II systematically overestimates the experimental values, particularly for methane flames. However, it successfully predicts a 

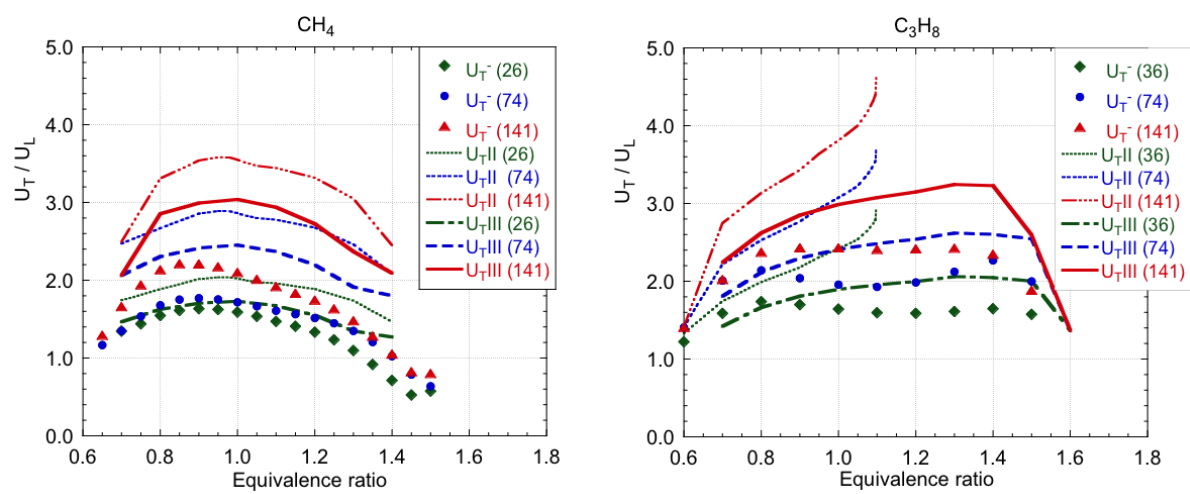

Fig. 8 Normalised self-turbulent flame speed of methane- (left) and propane- (right) air flames. The numerical values in the legend give the tube diameter in mm. Symbols: experimental values; tiny lines: Propagation speeds calculated using $\Lambda_{m}$ and $\lambda_{c}$ from Ma II [30, 31]; wide lines: $\Lambda_{m}$ and $\lambda_{c}$ from Ma III [28]

velocity maximum on the lean side of methane-air flames. For propane flames, the curves are truncated beyond $\phi \approx 1$ because all richer propane-air flames were systematically unstable and thus it is not possible to obtain a Markstein number or a cut-off wavelength from the threshold of stability.

The values of Ma III [28] are higher than Ma II, leading to a larger cut-off wavelength. The velocity maximum on the lean side is less pronounced, but the calculated values of self-similar flame speed are closer to experimental results.

In fact, as the largest length scale is generally the diameter of the burner, these curves reproduce the inverse tendency of the cut-off wavelengths of both fuels, see figure 9. Such measurements of intrinsically unstable flames speed in tubes of different diameters could thus be used to determine the characteristic cut-off length-scale of combustible mixtures in a simpler way than by measuring the onset of instability on planar flames or the Markstein number on expanding spherical flames. However, the calculated increase of the flame speed with the tube diameter is still larger than that observed experimentally, particularly for methane-air flames. We have used results of the linear theory of flame stability to cal- 


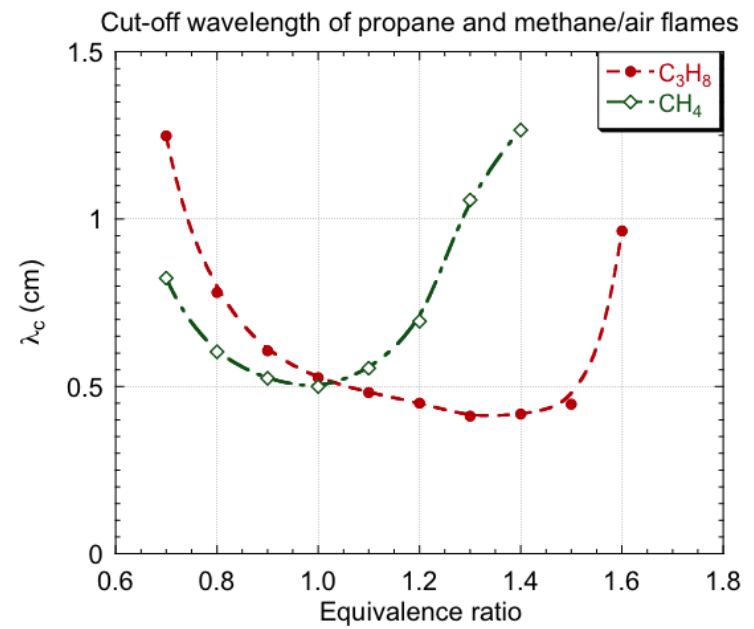

Fig. 9 Cut-off wavelengths for propane- and methane-air flames.

culate the largest possible flame scale and we generally obtain values much larger than the tube diameter. The largest cells should thus have the dimension of the tube diameter. This prediction is not confirmed by visual observation of the flames, particularly for the case of rich propane-air flames (see fig. 6) where the maximum cell size seems to be approximately $1 / 3$ of the tube diameter. This observation implies that relation (2) overestimates the self turbulent flame speed.

A better characterisation of the flame geometry is probably needed to determine characteristic length scales. Nevertheless, there is sufficient agreements between theory and experiment to support the influence of cut-off wavelengths on the speed of flame propagation in wide tubes. 


\section{Conclusion}

The propagation velocities of self-turbulent premixed flames propagating in quiescent mixtures were measured in tubes having diameters ranging from 26 to $141 \mathrm{~mm}$ in order to test the assumption of a self-similar behaviour in wide tubes.

An unexpected and striking result is that the free flame can propagate with different velocities in a given configuration, depending on the angle of tilt of the front with respect to the burner axis. Propagation velocities as high as $3.5 U_{L}$ were measured. This could be a relevant result for determining the limits of flash-back.

Supposing that the minimum value of the measured flame velocity is close to the fundamental self-turbulent flame speed, the experimental results were compared to a simple model equation for the propagation speed of cellular flames using two different sets of Markstein numbers. There is a reasonable qualitative agreement, and the calculated cut-off wavelengths explain the differences observed between rich methane- and propane-air flames. However, the predicted values of propagation velocity are generally significantly larger than the measured values. This difference is probably related to an overestimation of the largest characteristic lengths of the flame as evidenced by the topology of rich propane-air flames (fig. 6) and, we may also question the validity of determining cut-off wavelengths from a linear model of stability of planar flames. Moreover, the fractal exponent $D \approx 1 / 3$ was determined from experiments on large freely expanding spherical laminar flames. There is no solid argument to justify that flames in tubes will have exactly the same fractal exponent than freely expanding spherical flames, so the agreement is surprisingly good. 
Appendix - The acoustic damper

The purpose of the acoustic damper is to eliminate thermo-acoustic instabilities by absorbing acoustic perturbations arriving at the base of the tube. This is done by introducing a viscous loss (real acoustic impedance) at the base of the tube equal to the acoustic impedance of the gas in the tube. When this condition is fulfilled, propagating acoustic perturbations are dissipated at the base and not reflected. The principle is a transposition of that used in electronic transmission lines (e.g. 50 and $75 \mathrm{ohm}$ cables) where reflection of the signal is eliminated by terminating the line with a real resistance whose value is equal to the impedance of the cable.

The characteristic impedance of the gas in the tube, $Z=p^{\prime} / u^{\prime}$, is equal to i $\rho c$, where $p^{\prime}$, $u^{\prime}$ are the respectively acoustic pressure and displacement velocity, $\rho$ is the density of the gas, and $c$ is the speed of sound. For the mixtures used here, this impedance is very close to that of free air and has a value approximately equal to $410 \mathrm{~Pa} . \mathrm{s} / \mathrm{m}$. The pressure $p^{\prime}$ and the velocity $u^{\prime}$ are in phase quadrature, so the impedance is imaginary and there is negligible energy dissipation.

A thin annular slit of height $h$ and length $l$ is introduced at the base of the tube, see figure 1. An acoustic pressure perturbation $p^{\prime}$ introduces flow through the slit with an unsteady velocity $u_{s}$. The amplitude and phase of this flow will be determined by the viscous resistance to flow in the slit (in-phase or real component) and by the inertia of the gas in the slit (phase quadrature, or imaginary component)

The acoustically induced flow velocity in the annular slit is higher than the acoustic displacement velocity at the base of the tube. If the tube diameter is small compared to the acoustic wavelength, mass conservation imposes that the mean acoustic velocity $u^{\prime}$ at the base of the tube (diameter $\left.\varnothing=D_{0}\right)$ and the mean flow velocity $u_{s}$ in the annular slit $(\varnothing=$ 
$\left.D_{1}\right)$ are related by

$$
u_{S}(t) \pi D_{1} h=u^{\prime}(t) \frac{\pi D_{0}^{2}}{4}
$$

Assuming a Poiseuille flow in the slit, the mean velocity $u_{s}$ is just $2 / 3$ the maximum velocity, $u_{s}=2 / 3 u_{\max }$, and the viscous contribution to the instantaneous pressure drop across the slit, $p^{\prime}(t)=\hat{p} \cos (\omega t)$, is then given by [38]

$$
\hat{p} \cos (\omega t)=\frac{12 \mu l}{h^{2}} \hat{u}_{s} \cos (\omega t)
$$

where $\mu$ is the shear viscosity and $p^{\prime} / l$ is the pressure gradient across the slit. Since the flow is unsteady, there is also a contribution arising from the inertia of the fluid in the slit. The mass of fluid in the slit is $m=\rho \pi D_{1} h l$ and its instantaneous acceleration is $-\omega u_{s} \sin (\omega t)$. Equating the total force on the gas in the annular slit to the unsteady force required to overcome the viscous and inertial resistance of the flow, and in the approximation that the inertial contribution is small, we obtain

$$
\hat{p} \cos (\omega t)=\frac{12 \mu l}{h^{2}} \hat{u}_{S} \cos (\omega t)-\rho l \omega \hat{u}_{s} \sin (\omega t)
$$

where $u^{\prime}$ can be substituted for $u_{s}$ from equ. (9) to give the acoustic impedance:

$$
Z=\frac{p^{\prime}}{u^{\prime}}=\frac{D_{0}^{2} l}{h D_{1}}\left(\frac{3 \mu}{h^{2}}-\mathrm{i} \omega \frac{\rho}{4}\right)
$$

Equating the real part of the impedance at the bottom of the tube, $\operatorname{Re}[Z]$, to the imaginary part of the impedance of air, $\rho \mathrm{c}$, leads to the relation:

$$
h=\left(\frac{3 \mu D_{0}^{2} l}{\rho c D_{1}}\right)^{1 / 3}
$$

For the acoustic damper to be effective, the imaginary part of the impedance of the slit (inertial contribution) must be negligible compared to the real part, i.e.

$$
\frac{|\operatorname{Im}[Z]|}{|\operatorname{Re}[Z]|} \ll 1,
$$


which implies

$$
h \ll\left(\frac{12 \mu}{\rho \omega}\right)^{1 / 2},
$$

and from equ. (13) this also imposes

$$
l \ll \frac{8 c D_{1}}{D_{0}^{2}} \sqrt{\frac{3 \mu}{\rho \omega^{3}}} .
$$

Relation (16) imposes that the length of slit be relatively short, $l \approx 1 \mathrm{~mm}$, and according to (13), the height of slit has to be tuned to a value that is a function of both the slit length $l$ and the tube diameter, $D_{0}$. Typically $h \approx 0.2 \mathrm{~mm}$. Despite the short dimensions of the slit, the Poiseuille approximation appears to be sufficient and this device has proved to be very efficient in suppressing the thermo-acoustic instability otherwise encountered with premixed flames propagating in tubes.

\section{References}

1. E.E. Mallard and H. Le Chatelier. Recherches expérimentales et théoriques sur la combustion des mélanges gazeux explosifs. Annales des Mines, Paris, Series 8(4):274-377, 1883.

2. P. Clavin and F.A. Williams. Theory of premixed-flame propagation in large-scale turbulence. Journal of Fluid Mechanics, 90 part 3:589-604, 1979.

3. A. N. Lipatnikov and J. Chomiak. Turbulent flame speed and thickness: phenomenology, evaluation, and application in multi-dimensional simulations. Progress in Energy and Combustion Science, 28(1):1-74, 2002.

4. V.R. Savarianandama and C.J. Lawn. Burning velocity of premixed turbulent flames in the weakly wrinkled regime. Combustion and Flame, 146(1-2):1-18, July 2006. 
5. G. Darrieus. Propagation d'un front de flamme. Unpublished work presented at La Technique Moderne (1938), and at Le Congrès de Mécanique Appliquée (1945), 1938.

6. L. Landau. On the theory of slow combustion. Acta Physicochimica URSS, 19:77-85, 1944.

7. H. Guénoche and M. Jouy. Changes in the shape of flames propagating in tubes. In The Combustion Institute, editor, IVth International Symposium on Combustion, number 403-407, 1952.

8. P. Cambray and G. Joulin. On a scaling law for coarsening cells of premixed flames: an approach to fractalization. Combustion Science and Technology, 161:139-164, 2000.

9. Bychkov V. Importance of the darrieus-landau instability for strongly corrugated turbulent flames. Physical Review E, 68(6):art. 066304, Dec. 2003.

10. V. Akkerman, V. Bychkov, and L.E. Eriksson. Numerical study of turbulent flame velocity. Combustion and Flame, 151(4):452-471, 2007.

11. Filtayev S.A., Driscoll J.F., Carter C.D., and Donbar J.M. Measured properties of turbulent premixed flames for model assessment, including burning velocities, stretch rates, and surface densities. Combustion and Flame, 141:1-21, 2005.

12. O. Yu. Travnikov, V. V. Bychkov, and M. A. Liberman. Numerical studies of flames in wide tubes: Stability limits of curved stationary flames. Physical Review E, 61(1): $468-474,2000$.

13. Michael A. Liberman, Mikhail F. Ivanov, Oleg E. Peil, Damir M. Valiev, and Lars-Erik Eriksson. Numerical studies of curved stationary flames in wide tubes. Combustion Theory and Modelling, 7(4):653-676, October 2003. 
14. V. Akkerman and V. Bychkov. Turbulent flame and the Darrieus-Landau instability in a three-dimensional flow. Combustion Theory and Modelling, 7(4):767-794, November 2003.

15. V'yacheslav Akkerman and Vitaly Bychkov. Velocity of weakly turbulent flames of finite thickness. Combustion Theory and Modelling, 9(2):323-351, 2005.

16. B. Denet and V. Bychkov. Low vorticity and small gas expansion in premixed flames. Combustion Science and Technology, 177(8):1543-1566, August 2005.

17. Yu. A. Gostintsev, A. G. Istratov, and Yu. V. Shulenin. Self-similar propagation of a free turbulent flame in mixed gas mixtures. Combustion, Explosions and Shock Waves, 24(5):563-569, September 1988.

18. D. Bradley, T. M. Cresswell, and J. S. Puttock. Flame acceleration due to flame-induced instabilities in large-scale explosions. Combustion and Flame, 124(4):551-559, March 2001.

19. H.F. Coward and F.J. Hartwell. Studies in the mechanism of flame movement. Part I, the uniform movement of flame in mixtures of methane and air, in relation to tube diameter. Journal of the Chemical Society, (paper 277):1996-2004, 1932.

20. G. Searby. Acoustic instability in premixed flames. Combustion Science and Technology, 81:221-231, 1992.

21. G. Searby and D. Rochwerger. A parametric acoustic instability in premixed flames. Journal of Fluid Mechanics, 231:529-543, 1991.

22. B. Denet. Stationary solutions and Neumann boundary conditions in the Sivashinsky equation. Physical Review E, 74:036303, 2006. 
23. G. Joulin, B. Denet, and H. El-Rabii. Potential-flow models for channelled twodimensional premixed flames around near-circular obstacles. Physical Review E, 81 (1):016314, January 2010.

24. V. Bychkov and A. Kleev. The nonlinear equation for curved flames applied to the problem of flames in cylindrical tubes. Physics of Fluids, 11(7):1890-1895, July 1999.

25. K.J. Bosschaart and L.P.H. De Goey. The laminar burning velocity of flames propagating in mixtures of hydrocarbons and air measured with the heat flux method. Combustion and Flame, 136:264-269, 2004.

26. Zeldovich Y.B., Istratov A.G., Kidin N.I., and Librovich V.B. Flame propagation in tubes hydrodynamics and stability. Combustion Science and Technology, 24(1-2):1-13, 1980.

27. G. Searby and J. Quinard. Direct and indirect measurements of Markstein numbers of premixed flames. Combustion and Flame, 82(3-4):298-311, 1990.

28. S.G. Davis, J. Quinard, and G. Searby. Markstein numbers in counterflow, methaneand propane-air flames: a computational study. Combustion and Flame, 130:123-136, 2002.

29. P. Pelcé and P. Clavin. Influence of hydrodynamics and diffusion upon the stability limits of laminar premixed flames. Journal of Fluid Mechanics, 124:219-237, 1982.

30. J. Quinard, G. Searby, and L. Boyer. Stability limits and critical size of structures in premixed flames. Progress in Astronautics and Aeronautics, 95:129-141, 1985.

31. Joël Quinard. Limites de stabilité et structures cellulaires dans les flammes de prémélange. PhD Thesis, Université de Provence, Marseille, December 1984. 
32. P. Clavin and P. Garcia. The influence of the temperature dependence of diffusivities on the dynamics of flame fronts. Journal de Mécanique Théorique et Appliquée, 2(2): 245-263, 1983.

33. Chris Morley. Gaseq a chemical equilibrium program for windows, 2005. URL http://www.gaseq.co.uk/.

34. D. Bradley, P. H. Gaskell, and X. J. Gu. Burning velocities, (m)arkstein lengths, and flame quenching for spherical methane-air flames: A computational study. Combustion and Flame, 104(1-2):176-198, January 1996.

35. D. Bradley, R.A. Hicks, M. Lawes, C.G.W. Sheppard, and R. Woolley. The measurement of laminar burning velocities and Markstein numbers for iso-octane and isooctane-n-heptane- air mixtures at elevated temperatures and pressures in an explosion bomb. Combustion and Flame, 115:126-144, 1998.

36. X. J. Gu, M. Z. Haq, M. Lawes, , and R. Woolley. Laminar burning velocity and Markstein lengths of methane-air mixtures. Combustion and Flame, 121(1-2):41-58, 2000.

37. G. J. Sharpe. Effect of thermal expansion on the linear stability of planar premixed flames for a simple chain-branching model: The high activation energy asymptotic limit. Combustion Theory and Modelling, 12(4):717-738, August 2008.

38. Frank M. White. Fluid Mechanics. Mc Graw Hill, 3rd edition, 1994. 